\title{
Original Research \\ Comparison of NICE and ESC strategy for risk assessment in women with stable chest pain: a coronary computed tomography angiography study
}

\author{
Hantao Jiang ${ }^{1, \dagger}$, Chao Feng ${ }^{1, \dagger}$, Ying Jin ${ }^{1}$, Jinping Feng ${ }^{1}$, Guangsheng Li $^{2}$, Pei Ren ${ }^{1}$, \\ Kai Ren ${ }^{1}$, Jia Zhou ${ }^{1, *}$ \\ ${ }^{1}$ Department of Cardiology, Tianjin Chest Hospital, 300000 Tianjin, China \\ ${ }^{2}$ Department of Pneumology, Tianjin Chest Hospital, 300000 Tianjin, China \\ *Correspondence: zhoujiawenzhang@126.com (Jia Zhou) \\ ${ }^{\dagger}$ These authors contributed equally. \\ Academic Editors: Brian Tomlinson and Takatoshi Kasai \\ Submitted: 13 November 2021 Revised: 1 December 2021 Accepted: 8 December 2021 Published: 17 January 2022
}

\begin{abstract}
Background: For women presenting with stable chest pain (SCP), the appropriate risk assessment strategy to identify individuals unlikely to benefit from further cardiovascular imaging testing (CIT) is debatable. Thus, the present study intended to compare two risk assessment strategies in these individuals. Methods: 2592 women with SCP who underwent coronary computed tomography angiography (CCTA) were divided into low and high risk group according to 2016 National Institute of Health and Care Excellence guideline-determined strategy (NICE strategy) and 2019 European Society of Cardiology guideline-determined strategy (ESC strategy), respectively. The associations of coronary artery disease (CAD), major adverse cardiovascular event (MACE) and other subsequent clinical outcomes with risk groups and net reclassification improvement (NRI) were evaluated to compare different strategies. Results: Both NICE strategy which focused on symptom evaluation and ESC strategy which was based on pretest probability (PTP) determined by ESC-PTP model and coronary artery calcium score-weighted clinical likelihood (CACS-CL) model classified a proportion ( $34.49 \%$ and $63.97 \%$, respectively) of individuals into the low risk group. Compared to NICE strategy, ESC strategy indicated stronger associations between risk groups and obstructive CAD (odds ratio: 27.63 versus 3.57), MACE (hazard ratios: 4.24 versus 1.91), more intensive clinical management as well as a positive NRI $(27.71 \%, p<0.0001)$. Conclusion: Compared to NICE strategy, ESC strategy which sequentially incorporated ESC-PTP model with CACS-CL model seemed to be associated with greater effectiveness in identifying individuals who may derive maximum benefit from further CIT in women presenting with SCP.
\end{abstract}

Keywords: Risk assessment strategy; Stable chest pain; Coronary artery calcium score; Women; Pretest probability; Coronary computed tomography angiography

\section{Introduction}

Coronary artery disease (CAD) is a previously underestimated cause of morbidity and mortality in women $[1,2]$. Major differences exist in the risk factors and clinical presentation in women compared to men [3,4], which influence designing an effective risk assessment strategy and evaluating the potential referral to cardiovascular imaging testing (CIT) $[5,6]$. Recently, data from four large CIT-based clinical trials demonstrated the discrepancy among high burden of traditional risk factors, atypical symptom and low prevalence of obstructive CAD, highlighting the need for optimal strateges to the evaluation and diagnosis of obstructive CAD in women presenting with stable chest pain (SCP) [710].

The 2016 U.K. National Institute of Health and Care Excellence (NICE) guideline offered a strategy recommending coronary computed tomography angiography (CCTA) for all individuals with typical and atypical angina or abnormal electrocardiogram (ECG) [11]. However, this symptom-based risk assessment strategy for SCP has been controversial since release [12-14] and numerous studies has indicated that atypical symptoms were more likely to be a manifestation of SCP in women [3,4,7-10]. Separately, the 2019 European Society of Cardiology (ESC) guideline advocated an updated pretest probability (PTP) model and recommended CIT for individuals with high ESC-PTP [15]. For individuals with borderline ESC-PTP, a new concept of clinical likelihood (CL) incorporating risk factors of CAD other than age, sex, and type of SCP was introduced [15] and Winther et al. [16] developed two models for the estimation of CL: risk factor-weighted CL (RF-CL) model and coronary artery calcium score (CACS)-weighted CL (CACS-CL) model.

2016 NICE guideline-determined risk assessment strategy (NICE strategy) [17,18], ESC-PTP model [19], RF-CL model and CACS-CL model [16] has been externally validated in general SCP individuals. But to date, no comparative analysis has been conducted to systematically evaluate NICE strategy and 2019 ESC guideline- 
Table 1. Characteristics by risk groups based on different strategies.

\begin{tabular}{|c|c|c|c|c|c|c|c|}
\hline & \multirow[t]{2}{*}{ Total } & \multicolumn{2}{|c|}{ NICE strategy } & \multicolumn{4}{|c|}{ ESC strategy } \\
\hline & & Low & High & $p$ & Low & High & $p$ \\
\hline & $\mathrm{n}=2592$ & $\mathrm{n}=894$ & $\mathrm{n}=1698$ & & $\mathrm{n}=1658$ & $\mathrm{n}=934$ & \\
\hline $\operatorname{Age}^{a}$ & $58.2 \pm 10.8$ & $56.9 \pm 11.7$ & $58.9 \pm 10.9$ & $<0.0001$ & $56.8 \pm 10.7$ & $60.7 \pm 11.3$ & $<0.0001$ \\
\hline Diabetes & $570(22)$ & $172(19)$ & $398(23)$ & 0.0162 & $298(18)$ & $272(29)$ & $<0.0001$ \\
\hline Hypertension & $1166(45)$ & $375(42)$ & $791(47)$ & 0.0268 & $680(41)$ & $487(52)$ & $<0.0001$ \\
\hline Hyperlipidemia & $829(32)$ & $250(28)$ & $579(34)$ & 0.0017 & $448(27)$ & $382(41)$ & $<0.0001$ \\
\hline Smoking & $441(17)$ & $125(14)$ & $316(19)$ & 0.0034 & $199(12)$ & $242(26)$ & $<0.0001$ \\
\hline Family history & $881(34)$ & $286(32)$ & $595(35)$ & 0.1298 & $514(31)$ & $367(39)$ & $<0.0001$ \\
\hline Abnormal ECG & $752(29)$ & $0(0)$ & $752(44)$ & $<0.0001$ & $448(27)$ & $304(32)$ & $<0.0001$ \\
\hline Symptom & & & & $<0.0001$ & & & $<0.0001$ \\
\hline Nonanginal chest pain & $934(36)$ & $894(100)$ & $40(2)$ & & $746(45)$ & $188(20)$ & \\
\hline Atypical anginal & $1270(49)$ & $0(0)$ & $1270(75)$ & & $812(49)$ & 458 (49) & \\
\hline Typical anginal & $388(15)$ & $0(0)$ & $388(23)$ & & $99(6)$ & $289(31)$ & \\
\hline $\mathrm{CACS}^{b}$ & $3(0-79)$ & $2(0-48)$ & $14(0-175)$ & $<0.0001$ & $0(0-25)$ & $31(3-254)$ & $<0.0001$ \\
\hline
\end{tabular}

Values are presented as $\mathrm{n}(\%)$ unless stated otherwise.

NICE strategy, 2016 National Institute of Health and Care Excellence guideline-determined risk assessment strategy;

ESC strategy, 2019 European Society of Cardiology guideline-determined risk assessment strategy; ECG, electro-

cardiogram.

${ }^{a}$ years, mean \pm standard deviation.

${ }^{b}$ median (25th-75th).

determined risk assessment strategy (ESC strategy) in women with SCP, for whom the appropriate decisionmaking of CIT was important but difficult $[5,6]$. Thus, we aimed to compare the effectiveness of two newest risk assessment strategies to optimize downstream clinical management in a CCTA-based cohort comprised of women presenting with SCP.

\section{Methods}

\subsection{Study population}

In Tianjin Chest Hospital, 5289 individuals referred to CCTA for assessment of SCP indicative of obstructive CAD were included from December 2015 to December 2017, and details about the total cohort were presented as previously described [20-22]. In this subgroup analysis, 2592 women were included and followed up until December 2019.

\subsection{Baseline data and risk assessment strategies}

Baseline data including age, hypertension, hyperlipidemia, smoking, abnormal ECG and symptom were collected as described previously [20-22]. SCP symptom were categorized as nonanginal chest pain, atypical angina, or typical angina [23].

Based on each strategy, CIT should be not referred for an individual at low risk. Details of risk groups in NICE and ESC strategy were as follows $[11,15]$ :

NICE strategy: Individuals with nonanginal SCP and normal ECG were divided into low risk group. Individuals with typical and atypical angina or nonanginal SCP with abnormal ECG were divided into high risk group [11].

ESC strategy: PTP of obstructive CAD was determined according to the ESC-PTP model based on age, sex and symptom [15]. Individuals with ESC-PTP $<5 \%$ were divided into low risk group and individuals with ESC-PTP $>15 \%$ were divided into high risk group. For individuals with ESC-PTP between 5\% and 15\%, we selected CACSCL model incorporating clinical variables plus CACS [16]. Based on the data from original study of CACS-CL model, CACS-CL $<15 \%$ was associated with a low prevalence of obstructive CAD $(<5 \%)$. Thus, individuals with ESC-PTP between $5 \%$ and $15 \%$ and CACS-CL $<15 \%$ were divided into low risk group and individuals with ESC-PTP between $5 \%$ and $15 \%$ and CACS-CL model $>15 \%$ were divided into high risk group.

For further investigation of the impact of CACS, we also used RF-CL model which had the same clinical variables as CACS-CL model [16] to classify individuals with ESC-PTP of 5-15\%.

\subsection{CACS and CCTA}

The imaging data collection of CACS and CCTA were conducted as described previously [20-22,24]. Obstructive $\mathrm{CAD}$ was defined as present if an individual had at least one lesion with $\geq 50 \%$ diameter stenosis or any unassessable segments at CCTA. A positive individual was defined as an individual who had obstructive CAD. 


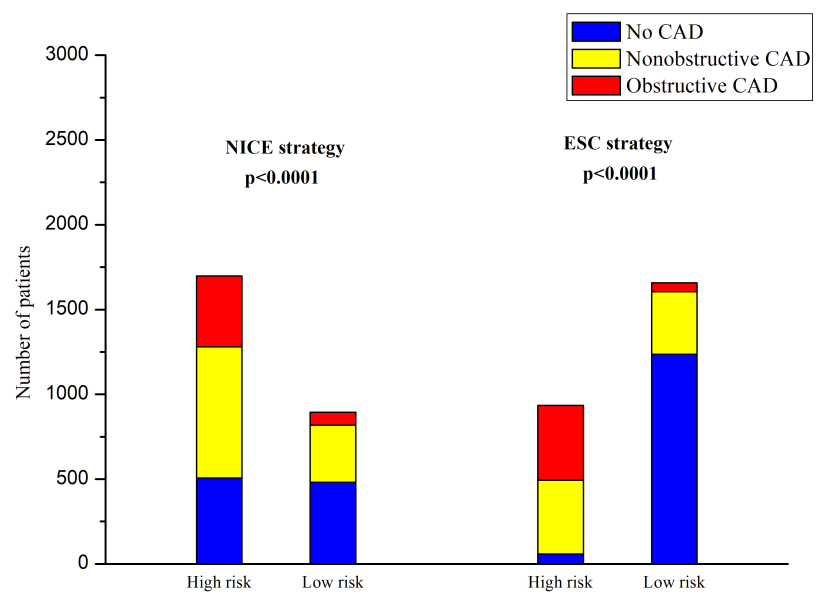

Fig. 1. Distribution of CAD in different risk groups. NICE strategy, 2016 National Institute of Health and Care Excellence guideline-determined risk assessment strategy; ESC strategy, 2019 European Society of Cardiology guideline-determined risk assessment strategy; CAD, coronary artery disease.

\subsection{Follow up and clinical events}

After CCTA, all individuals were followed at 6, 12, 24,36 and 48 months by phone call or physician visit. The primary endpoint was major adverse cardiovascular events (MACE) and it was defined as cardiac death and myocardial infarction. Cardiac death was defined as any death caused by cardiac disease or for which no other cause could be found. Myocardial infarction was defined according to the Fourth Universal Definition of Myocardial Infarction [25]. The changes of downstream clinical management within 60 days after CCTA were identified on electronic medical system and the secondary endpoint included increase of medication (IM), invasive coronary angiography (ICA) and coronary revascularization (CR). All endpoints were adjudicated via review of follow-up information and medical records by an independent clinical event committee who were blinded to other data.

\subsection{Statistical analysis}

R (version 3.2.4; R Foundation for Statistical Computing, Vienna, Austria) and MedCalc (version 15.2.2; MedCalc Software, Mariakerke, Belgium) were used for all statistical analyses. Student's $t$-test and Mann Whitney U-test were used to compare differences for continuous data as appropriate. Fisher exact test or $\chi^{2}$ test were used to compare categorical variables as appropriate. The reclassification table was used to calculate net reclassification improvement (NRI) which determined how a risk assessment strategy reclassified individuals compared with another [26]. Cox regression models were used to calculate hazard ratios (HRs) and $95 \%$ confidence intervals (CIs), which assessed association of risk groups to the time to the first MACE (or censoring). Kaplan-Meier curves were plotted for survival from
MACE and compared by log-rank test. All tests were wotailed and $p<0.05$ was statistically significant.

\section{Results}

\subsection{Baseline characteristics}

According to NICE strategy, of the 2592 women, $34.49 \%$ (894/2592) were divided into low risk group. Among 855 individuals with ESC-PTP of 5-15\%, 529 individuals had a CACS-CL $<15 \%$. Together with the 1129 individuals with ESC-PTP $<5 \%$, ESC strategy totally divided $63.97 \%$ (1658/2592) women into low risk group. Table 1 shows the distribution of clinical characteristics by risk groups based on two strategies. Except family history, differences of the other baseline characteristics were statistically significant between two risk groups determined by NICE strategy. Separately, all baseline characteristics had significant differences in terms of ESC strategy.

\subsection{Risk groups and CAD}

As shown in Fig. 1, no, nonobstructive and obstructive CAD was found on CCTA in 493, 804 and 1295 individuals, respectively. Compared to individuals in low risk group, individuals in high risk group had more obstructive CAD [NICE strategy: $8.39 \%$ (75/894) versus $24.62 \%$ (418/1698), odds ratio: 3.57, 95\% CI: 2.75-4.63, $p<$ 0.0001; ESC strategy: 47.22\% (441/934) versus 3.13\% (52/1658), odds ratio: $27.63,95 \%$ CI: 20.37-37.46, $p<$ $0.0001]$.

\subsection{Primary endpoint}

Individuals were followed for 25 (interquartile range: 20 to 32) months. During the follow-up, 87 individuals experienced MACE: 11 individuals died from cardiac cause and 76 individuals suffered from nonfatal MI. Fig. 2 illustrates Kaplan-Meier estimates of individuals surviving free from MACE. High risk group according to both NICE and ESC strategy had a significantly higher risk of MACE, respectively ( $p$ for log-rank test: 0.0194 for NICE strategy and 0.0001 for ESC strategy), but the association of ESC strategy-determined risk groups (high versus low) with MACE was stronger than that of NICE strategy (HR for NICE strategy: $1.78,95 \%$ CI: $1.09-2.90, p=0.0221$ and HR for ESC strategy: 5.10, 95\% CI: 3.15-8.26, $p<$ $0.0001)$.

\subsection{Secondary endpoints}

As shown in Fig. 3, 761 individuals were referred to ICA after CCTA and 163 individuals underwent CR. In both strategies, rates of three secondary endpoints were significantly ( $p<0.0001$ for all) higher in the high risk groups than those in the low risk groups. The association of CR to risk groups in ESC strategy was conspicuously strong (OR: 6.36, 95\% CI: 4.39-9.20, $p<0.0001)$. 

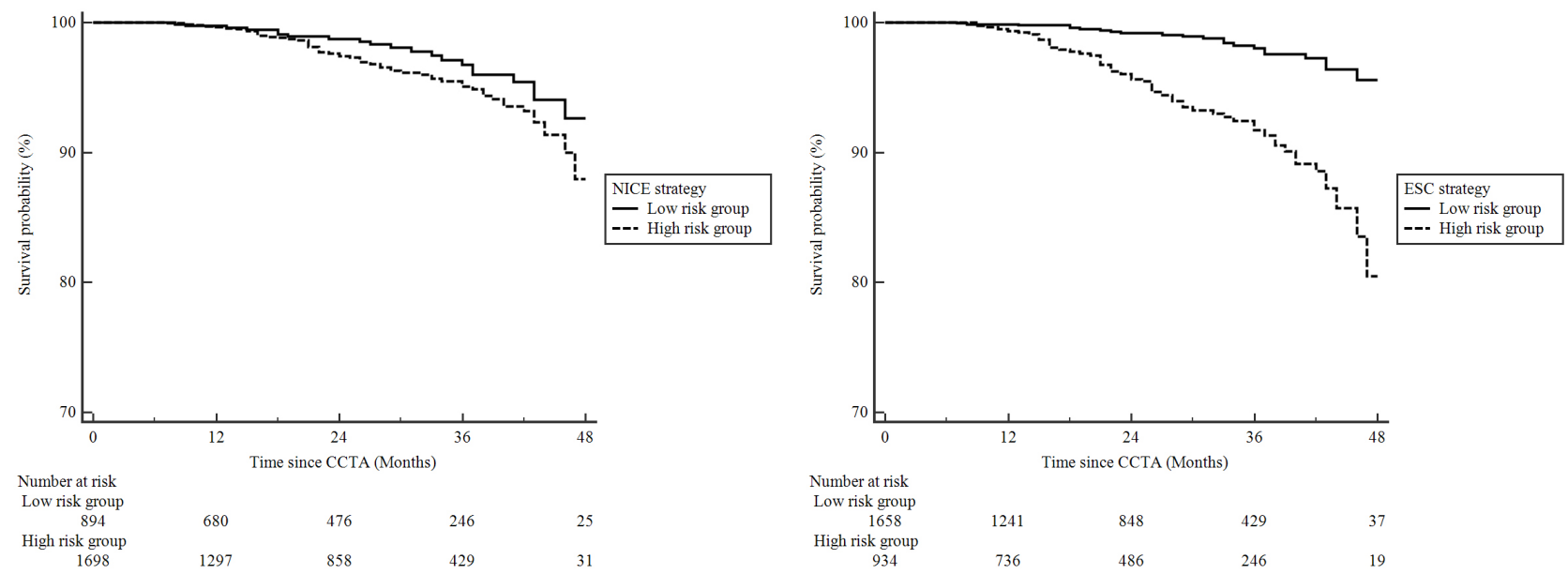

Fig. 2. Kaplan-Meier curves comparing high and low risk groups determined by NICE and ESC strategy. Abbreviations as in Fig. 1.

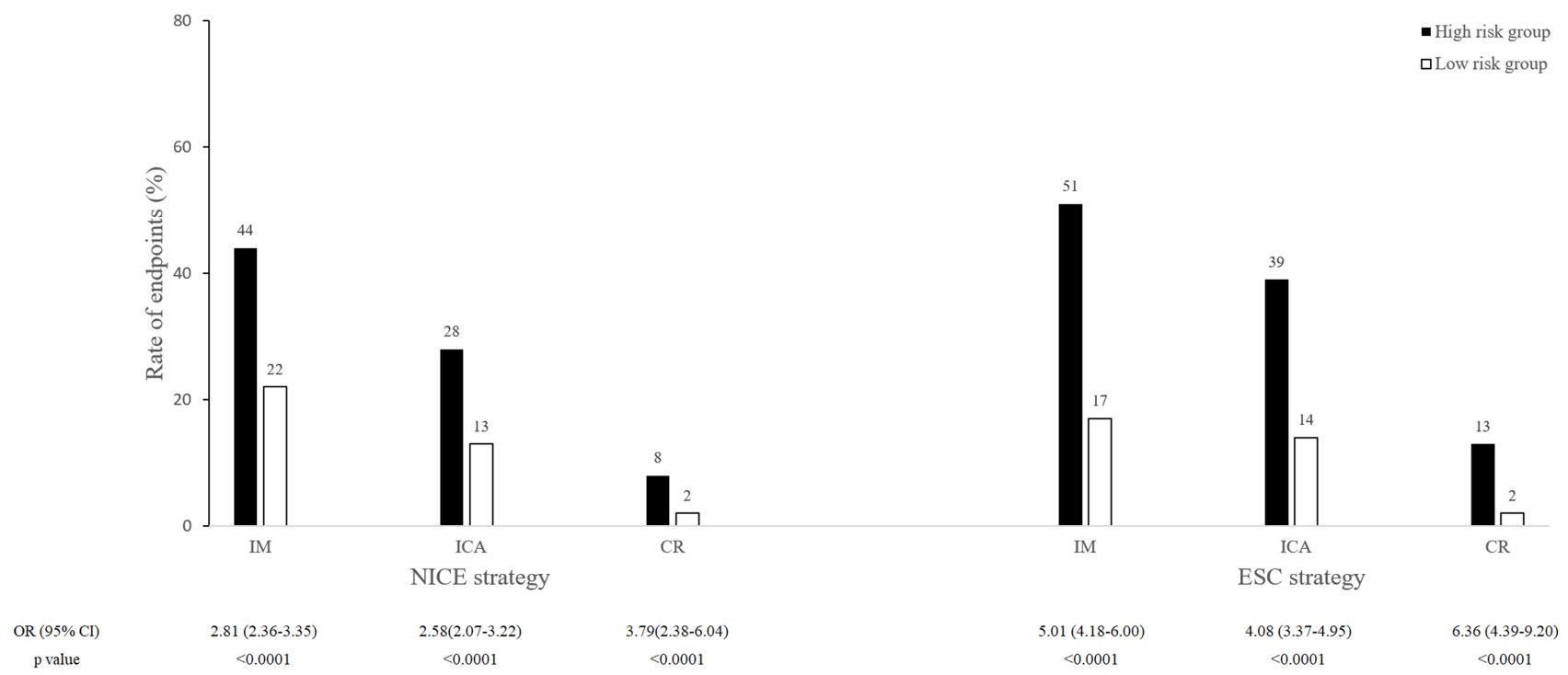

Fig. 3. Rates for secondary endpoints in low and high risk groups determined by NICE and ESC strategy. IM, Increase of medication; CR, coronary revascularization; ICA, invasive coronary angiography; OR, odds ratio; CI, confidence interval; Other abbreviations as in Fig. 1.

\subsection{Reclassification table and NRI}

In Table 2, compared to NICE strategy, ESC strategy correctly divided 843 from high to low risk, but 56 from low to high in the 2099 negative individuals. Of the 493 positive individuals, 36 were correctly divided to high risk but 13 to low. Thus, compared to NICE strategy, the NRI for ESC strategy was $37.49 \%$ for negative, $4.66 \%$ for positive, and $42.15 \%$ for all $(p<0.0001)$.

When replacing CACS-CL model with RF-CL model in individuals with borderline ESC-PTP and comparing ESC strategy (without CACS) to NICE strategy in Table 3, only 224 negative individuals were correctly reclassified into low risk by ESC strategy (without CACS) and the NRI was attenuated markedly $(7.05 \%$ in negative and $8.47 \%$ in all). Thus, comparing ESC strategy to NICE strategy in Table 2, the correct reclassification of 843 negative individuals should be in large measure $(73.43 \%, 619 / 843)$ attributed to the application of CACS in 855 individuals with borderline ESC-PTP. Moreover, only 1 of the 619 individuals suffered from nonfatal myocardial infarction and no individual died in the follow-up.

\section{Discussion}

In this CCTA-based cohort comprised of women with SCP, we demonstrated that based on current two risk assessment strategies, low risk groups were associated with fewer obstructive CAD, MACE and changes of downstream management than high risk groups did. Compared to NICE 
Table 2. Reclassification table comparing NICE and ESC strategy.

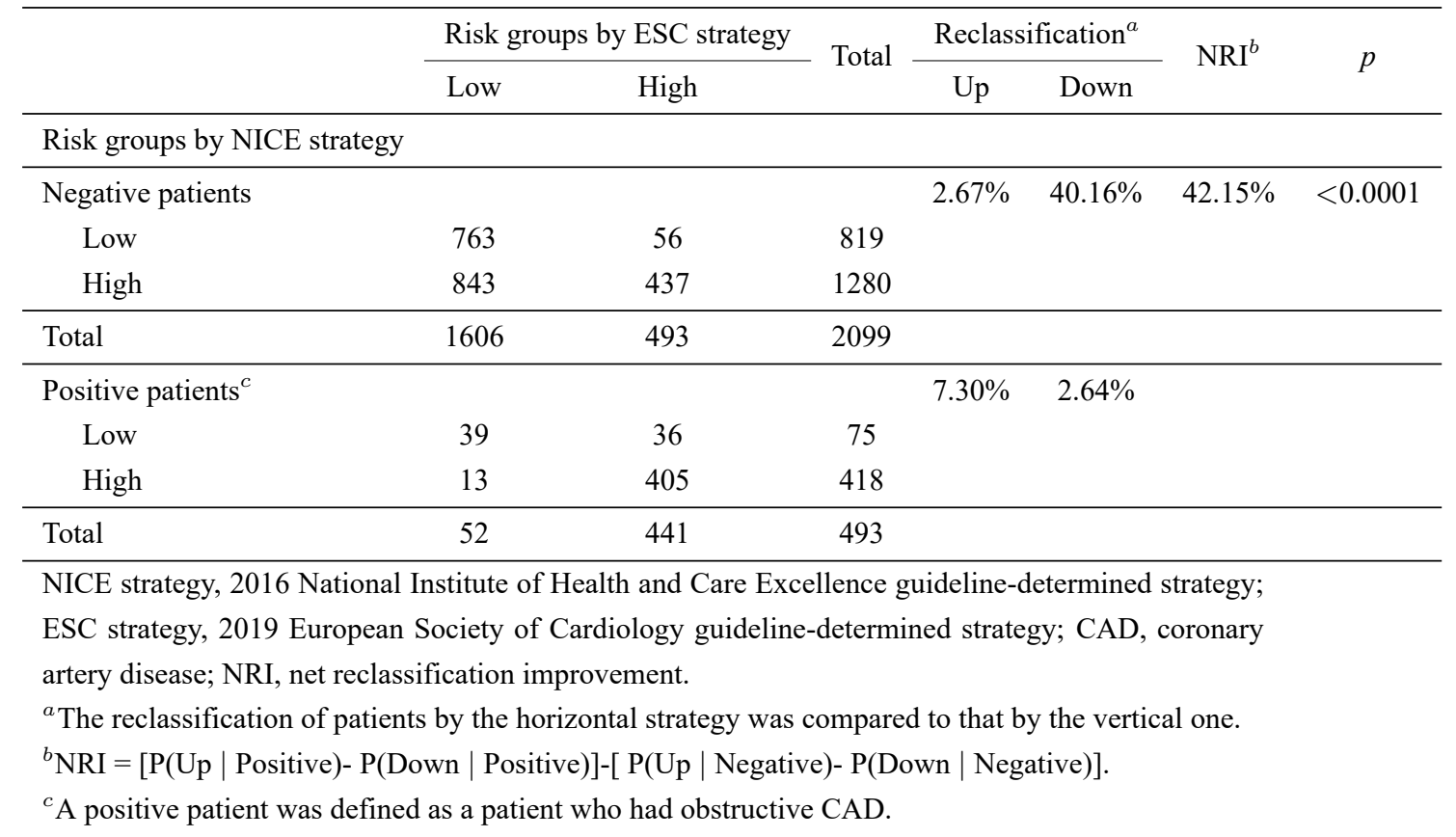

strategy which focused on symptom evaluation, ESC strategy seemed to be associated with greater effectiveness in identifying individuals at low risk. The superiority of ESC strategy could be mainly due to the application of CACS in individuals with borderline ESC-PTP, suggesting that a sequential instrument incorporating CACS with PTP estimation might offer effective deferral for CIT in women presenting with SCP.

In the present study, NICE strategy seemed to be associated with less effectiveness in identifying individuals at low risk compared to ESC strategy. It has been well established that women more often had traditional risk factors, atypical symptoms which might result from the low ability to exercise maximally and high prevalence of nonobstructive and small vessel disease, and lower rates of obstructive CAD than men [3,7]. In line with these, only $19.02 \%$ (493/2592) individuals had obstructive CAD detected on CCTA and nearly half $(49.00 \%, 1270 / 2592)$ reported atypical anginal in this study. Thus, although NICE strategy improved clinical outcomes compared to traditional strategies in general SCP individuals $[17,18]$, the suboptimal performance of NICE strategy may, to a large extent, be attributed to the insufficient power of symptom evaluation alone in women with SCP.

Among the new predictors of obstructive CAD, CACS has been proved to offer the most incremental information of diagnosis and prognosis above traditional cardiovascular risk factors [27-29]. Moreover, there was a significant increase in clinically useful reclassification when CACS was added to the risk assessment for women [30]. Thus, to permit more accurate risk assessment in women with a borderline ESC-PTP, we used CACS-CL model which incorporated clinical variables plus CACS and revealed the most robust determination of PTP among proposed models [16]. We also replaced CACS-CL model by RF-CL model which had the same clinical variables as CACS-CL model [16] and found that the additional application of CACS made a major contribution to the remarkably risk assessment improvement of ESC strategy. This paradigm incorporating CACS for borderline individuals showed paramount safety according to the extremely low likelihood $(3.14 \%, 52 / 1658)$ of obstructive CAD and rate $(1.33 \%, 22 / 1658)$ of cardiovascular events in low risk group. In this context, more emphasis should be placed on ESC strategy for the effective identification of women with SCP who may derive minimal benefit from further CIT in clinical practice. It also bears mentioning that ESC strategy would avoid unnecessary CIT at the expense of additional CACS scans. Thus, cost-effectiveness evaluation of this attractive risk assessment strategy for women with SCP is needed in the further.

\section{Limitations}

Although this is the first study to evaluate current risk assessment strategies for women with SCP, several issues merit consideration. First, this study was an observational cohort. Clinical management of individuals before and after CCTA were relied on local physician. Thus, whether ESC strategy will lead to more appropriate decision-making of downstream referral and better clinical outcomes for women with SCP need to be addressed in future studies, such as randomized controlled trials. Second, accumulating evidence support strategies which suggests widespread CACS screening $[8,30]$, such as the strategy applying CACS-CL to the entire SCP women [31]. Cost-effectiveness of this strategy warrant further evaluation. Third, using data from PROMISE cohort, Fordyce $e t$ 
Table 3. Reclassification table comparing ESC strategy (without CACS) and NICE strategy.

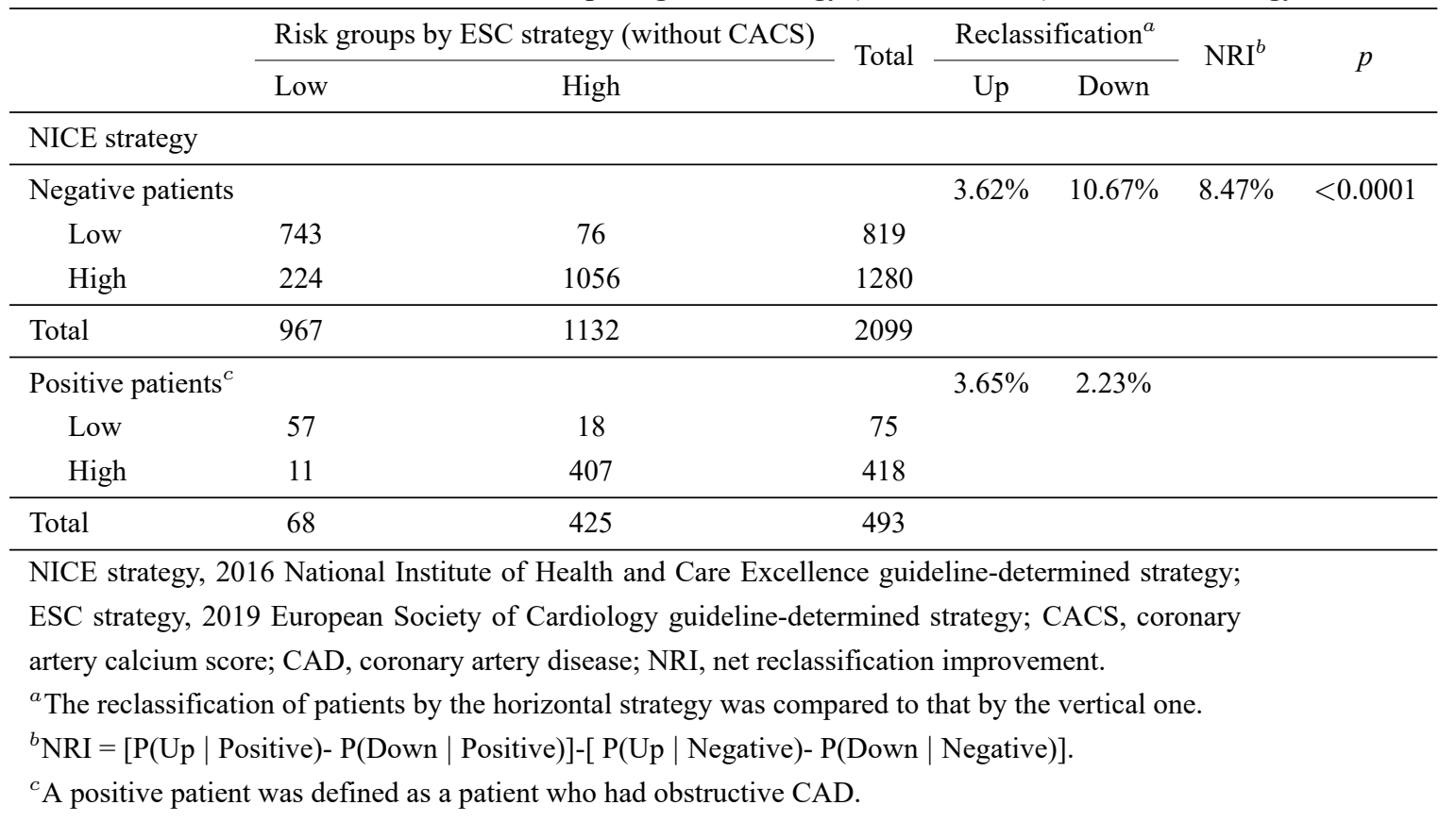

al. [32] developed a new tool to identify individuals deriving minimal value from CIT. Although the PROMISE minimal risk tool [33] has been externally validated, no recent clinical guideline recommends it as the risk assessment tool for individuals with SCP. Forth, this analysis focused on the presence of obstructive CAD documented by CCTA. Previous studies have demonstrated that CCTA had a high negative predictive value compared with invasive coronary angiogram $[34,35]$. So CCTA could offer robust reassurance for both strategies to exclude obstructive $\mathrm{CAD}$. Moreover, we defined unassessable segments as positive ones based on current guideline recommendations in which further testing should be referred for nonconclusive CCTA. Fifth, some female-specific risk factors, such as estrogen status and gestational diabetes mellitus, have shown the potential to improve risk assessment for women [36]. However, additional tests are needed for these attractive biomarkers and cost-effectiveness of them warrant further evaluation. Finally, the long-term follow-up information of secondary outcomes, such as compliance of medication were not collected.

\section{Conclusions}

Compared to symptom-focused strategy, ESC strategy which sequentially amalgamated CACS with PTP estimation might have more potential to effectively identify individuals who may derive minimal benefit from further CIT in women presenting with SCP potentially related to obstructive CAD. For more accurate and convenient risk assessment in these individuals, investigations with comprehensive and rigorous design are needed in the future.

\section{Author contributions}

$\mathrm{HJ}, \mathrm{CF}$ and $\mathrm{JZ}$ designed the study. HJ and CF wrote the manuscript. YJ, JF, GL, PR and KR collected data. JZ revised the manuscript and provided help and advice on the statistical analysis. YJ, JF, GL, PR and KR analyzed the data. All authors read and approved the final manuscript.

\section{Ethics approval and consent to participate}

All subjects gave their informed consent for inclusion before they participated in the study. The study was conducted in accordance with the Declaration of Helsinki, and the protocol was approved by the Ethics Committee of Tianjin Chest Hospital (approval number: 2017-KY-004).

\section{Acknowledgment}

We would like to express our gratitude to all those who helped us during the writing of this manuscript. Thanks to all the peer reviewers for their opinions and suggestions.

\section{Funding}

This study was supported by grants from Research Program of Tianjin Chest Hospital (2018XKC10).

\section{Conflict of interest}

The authors declare no conflict of interest.

\section{References}

[1] Aggarwal NR, Patel HN, Mehta LS, Sanghani RM, Lundberg GP, Lewis SJ, et al. Sex Differences in Ischemic Heart Disease: Advances, Obstacles, and Next Steps. Circulation: Cardiovascular Quality and Outcomes. 2018; 11: e004437

[2] Gupta A, Wang Y, Spertus JA, Geda M, Lorenze N, Nkonde- 
Price C, et al. Trends in Acute Myocardial Infarction in Young Patients and Differences by Sex and Race, 2001 to 2010. Journal of the American College of Cardiology. 2014; 64: 337-345.

[3] Mehta PK, Bess C, Elias-Smale S, Vaccarino V, Quyyumi A, Pepine CJ, et al. Gender in cardiovascular medicine: chest pain and coronary artery disease. European Heart Journal. 2019; 40: 3819-3826.

[4] Zhao M, Vaartjes I, Graham I, Grobbee D, Spiering W, KlipsteinGrobusch K, et al. Sex differences in risk factor management of coronary heart disease across three regions. Heart. 2017; 103: $1587-1594$.

[5] Bugiardini R, Yan AT, Yan RT, Fitchett D, Langer A, Manfrini $\mathrm{O}$, et al. Factors influencing underutilization of evidence-based therapies in women. European Heart Journal. 2011; 32: 1337 1344.

[6] Baldassarre LA, Raman SV, Min JK, Mieres JH, Gulati M, Wenger NK, et al. Noninvasive Imaging to Evaluate Women with Stable Ischemic Heart Disease. JACC: Cardiovascular Imaging. 2016; 9: 421-435.

[7] Hemal K, Pagidipati NJ, Coles A, Dolor RJ, Mark DB, Pellikka PA, et al. Sex Differences in Demographics, Risk Factors, Presentation, and Noninvasive Testing in Stable Outpatients with Suspected Coronary Artery Disease: Insights From the PROMISE Trial. JACC: Cardiovascular Imaging. 2016; 9: 337-346.

[8] Lubbers M, Coenen A, Bruning T, Galema T, Akkerhuis J, Krenning B, et al. Sex Differences in the Performance of Cardiac Computed Tomography Compared with Functional Testing in Evaluating Stable Chest Pain: Subanalysis of the Multicenter, Randomized CRESCENT Trial (Calcium Imaging and Selective CT Angiography in Comparison to Functional Testing for Suspected Coronary Artery Disease). Circulation: Cardiovascular Imaging. 2017; 10: e005295.

[9] Mangion K, Adamson PD, Williams MC, Hunter A, Pawade T, Shah ASV, et al. Sex associations and computed tomography coronary angiography-guided management in patients with stable chest pain. European Heart Journal. 2020; 41: 1337-1345.

[10] Reynolds HR, Shaw LJ, Min JK, Spertus JA, Chaitman BR, Berman DS, et al. Association of Sex With Severity of Coronary Artery Disease, Ischemia, and Symptom Burden in Patients With Moderate or Severe Ischemia: Secondary Analysis of the ISCHEMIA Randomized Clinical Trial. JAMA Cardiology. 2020; 5: $773-786$

[11] Timmis A, Roobottom CA. National Institute for Health and Care Excellence updates the stable chest pain guideline with radical changes to the diagnostic paradigm. Heart. 2017; 103: $982-$ 986.

[12] Cremer PC, Nissen SE. The National Institute for Health and Care Excellence update for stable chest pain: poorly reasoned and risky for patients. Heart. 2017; 103: 972-974.

[13] Udelson JE, Hoffmann U. The United Kingdom's National Institute for Health and Care Excellence guideline on chest pain of recent onset: a United States perspective. Journal of Nuclear Cardiology. 2017; 24: 1535-1539.

[14] Hecht HS, Shaw L, Chandrashekhar YS, Bax JJ, Narula J. Should NICE guidelines be universally accepted for the evaluation of stable coronary disease? A debate. European Heart Journal. 2019; 40: 1440-1453.

[15] Knuuti J, Wijns W, Saraste A, Capodanno D, Barbato E, FunckBrentano C, et al. 2019 ESC Guidelines for the diagnosis and management of chronic coronary syndromes. European Heart Journal. 2020; 41: 407-477

[16] Winther S, Schmidt SE, Mayrhofer T, Botker HE, Hoffmann U, Douglas PS, et al. Incorporating Coronary Calcification Into Pre-Test Assessment of the Likelihood of Coronary Artery Disease. Journal of the American College of Cardiology. 2020; 76 :
$2421-2432$

[17] Adamson PD, Newby DE, Hill CL, Coles A, Douglas PS, Fordyce CB. Comparison of International Guidelines for Assessment of Suspected Stable Angina: Insights From the PROMISE and SCOT-HEART. JACC: Cardiovascular Imaging. 2018; 11: 1301-1310.

[18] Adamson PD, Hunter A, Williams MC, Shah ASV, McAllister DA, Pawade TA, et al. Diagnostic and prognostic benefits of computed tomography coronary angiography using the $2016 \mathrm{Na}-$ tional Institute for Health and Care Excellence guidance within a randomised trial. Heart. 2018; 104: 207-214.

[19] Bing R, Singh T, Dweck MR, Mills NL, Williams MC, Adamson PD, et al. Validation of European Society of Cardiology pretest probabilities for obstructive coronary artery disease in suspected stable angina. European Heart Journal - Quality of Care and Clinical Outcomes. 2020; 6: 293-300.

[20] Zhou J, Chen Y, Zhang Y, Wang H, Tan Y, Liu Y, et al. Epicardial Fat Volume Improves the Prediction of Obstructive Coronary Artery Disease above Traditional Risk Factors and Coronary Calcium Score. Circulation: Cardiovascular Imaging. 2019; 12: e008002.

[21] Zhou J, Liu Y, Huang L, Tan Y, Li X, Zhang H, et al. Validation and comparison of four models to calculate pretest probability of obstructive coronary artery disease in a Chinese population: a coronary computed tomographic angiography study. Journal of Cardiovascular Computed Tomography. 2017; 11: 317-323.

[22] Zhang Y, Liu Y, Zhang H, Zhou J. Impact of sex-specific differences in calculating the pretest probability of obstructive coronary artery disease in symptomatic patients: a coronary computed tomographic angiography study. Coronary Artery Disease. 2019; 30: 124-130.

[23] Diamond GA, Forrester JS. Analysis of Probability as an Aid in the Clinical Diagnosis of Coronary-Artery Disease. New England Journal of Medicine. 1979; 300: 1350-1358.

[24] Leipsic J, Abbara S, Achenbach S, Cury R, Earls JP, Mancini GJ, et al. SCCT guidelines for the interpretation and reporting of coronary CT angiography: a report of the Society of Cardiovascular Computed Tomography Guidelines Committee. Journal of Cardiovascular Computed Tomography. 2014; 8: 342-358.

[25] Thygesen K, Alpert JS, Jaffe AS, Chaitman BR, Bax JJ, Morrow DA, et al. Fourth Universal Definition of Myocardial Infarction (2018). Journal of the American College of Cardiology. 2018; 72: 2231-2264

[26] Alba AC, Agoritsas T, Walsh M, Hanna S, Iorio A, Devereaux PJ, et al. Discrimination and Calibration of Clinical Prediction Models: Users' Guides to the Medical Literature. The Journal of the American Medical Association. 2017; 318: 1377.

[27] Genders TS, Steyerberg EW, Hunink MG, Nieman K, Galema TW, Mollet NR, et al. Prediction model to estimate presence of coronary artery disease: retrospective pooled analysis of existing cohorts. British Medical Journal. 2012; 344: e3485

[28] Genders TSS, Coles A, Hoffmann U, Patel MR, Mark DB, Lee KL, et al. The External Validity of Prediction Models for the Diagnosis of Obstructive Coronary Artery Disease in Patients With Stable Chest Pain: Insights From the PROMISE Trial. JACC Cardiovasc Imaging. 2018; 11: 437-446

[29] Mittal TK, Pottle A, Nicol E, Barbir M, Ariff B, Mirsadraee S, et al. Prevalence of obstructive coronary artery disease and prognosis in patients with stable symptoms and a zero-coronary calcium score. European Heart Journal - Cardiovascular Imaging. 2017; 18: 922-929.

[30] Nakao YM, Miyamoto Y, Higashi M, Noguchi T, Ohishi M, Kubota I, et al. Sex differences in impact of coronary artery calcification to predict coronary artery disease. Heart. 2018; 104: $1118-1124$.

[31] Zhou J, Li C, Cong H, Duan L, Wang H, Wang C, et al. Compar- 
ison of Different Investigation Strategies to Defer Cardiac Testing in Patients with Stable Chest Pain. JACC: Cardiovascular Imaging. 2021. (in press)

[32] Fordyce CB, Douglas PS, Roberts RS, Hoffmann U, Al-Khalidi HR, Patel MR, et al. Identification of Patients with Stable Chest Pain Deriving Minimal Value from Noninvasive Testing: The PROMISE Minimal-Risk Tool, A Secondary Analysis of a Randomized Clinical Trial. JAMA Cardiology. 2017; 2: 400.

[33] Adamson PD, Fordyce CB, McAllister DA, Udelson JE, Douglas PS, Newby DE. Identification of patients with stable chest pain deriving minimal value from coronary computed tomography angiography: an external validation of the PROMISE minimal-risk tool. International Journal of Cardiology. 2018; 252: $31-34$.

[34] Haase R, Schlattmann P, Gueret P, Andreini D, Pontone G,
Alkadhi H, et al. Diagnosis of obstructive coronary artery disease using computed tomography angiography in patients with stable chest pain depending on clinical probability and in clinically important subgroups: meta-analysis of individual patient data. British Medical Journal. 2019; 365: 11945

[35] Knuuti J, Ballo H, Juarez-Orozco LE, Saraste A, Kolh P, Rutjes AWS, et al. The performance of non-invasive tests to rule-in and rule-out significant coronary artery stenosis in patients with stable angina: a meta-analysis focused on post-test disease probability. European Heart Journal. 2018; 39: 3322-3330.

[36] Rademaker AA, Danad I, Groothuis JG, Heymans MW, Marcu $\mathrm{CB}$, Knaapen $\mathrm{P}$, et al. Comparison of different cardiac risk scores for coronary artery disease in symptomatic women: do female-specific risk factors matter? European Journal of Preventive Cardiology. 2014; 21: 1443-1450. 